\title{
Long-term functional alterations in sports concussion
}

\author{
Louis De Beaumont, Ph.D., ${ }^{1,2}$ Luke C. Henry, Ph.D., ${ }^{3}$ And Nadia Gosselin, Ph.D. ${ }^{1}$ \\ ${ }^{1}$ Montreal Sacred Heart Hospital Research Centre, Montreal; ${ }^{2}$ Department of Psychology, University of \\ Quebec at Trois-Rivières, Quebec, Canada; and ${ }^{3}$ University of Pittsburgh Medical Center, Pittsburgh, \\ Pennsylvania
}

\begin{abstract}
In this review the authors discuss persistent and cumulative alterations in both cognitive and motor function after sports concussions detected with some of the newest, most sophisticated brain investigation techniques. Ranging from subclinical neurophysiological alterations in young concussed athletes to quantifiable cognitive and motor function declines in former athletes in late adulthood with concussions sustained decades earlier, this review is also intended to provide new insights into the neuropathophysiology of sports concussion.
\end{abstract} (http://thejns.org/doi/abs/10.3171/2012.9.FOCUS12278)

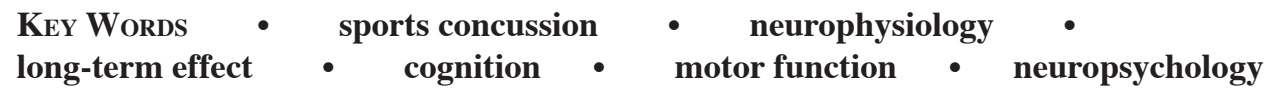

$\mathrm{T}$ HE prevailing attitude in sports culture was once rather dismissive of concussions, viewing them as minor injuries without consequence. ${ }^{71}$ This mindset has undergone a major overhaul, particularly in the last 10-20 years, and concussions are now taken much more seriously, in part because of the forced retirement of several high-profile athletes. The effects of a single concussion are rarely long-term owing to the spontaneous resolution of symptoms and better management strategies; however, long-term changes ranging from subtle electrophysiological alterations in cognitive func$\operatorname{tion}^{4,17,18,20,23,26,35,37,64,65}$ to persistent or more slowly resolving symptomatology $y^{11,24,27,41}$ as well as neurocognitive changes on reliable neuropsychological tests ${ }^{10,11,27,37,41}$ are detectible, particularly after multiple concussions. Furthermore, anatomical changes have also been documented. ${ }^{12,48,52}$ In this review, across a variety of subclinical and clinical findings pertaining to the spheres of cognition and motor function, we discuss the relevant differences observed in the chronic postinjury phase in active as well as retired athletes who have sustained multiple concussive blows.

\footnotetext{
Abbreviations used in this paper: $\mathrm{COP}=$ center of pressure; $\mathrm{CSP}$ $=$ cortical silent period; $\mathrm{ERP}=$ event-related potential; $\mathrm{GABA}_{\mathrm{B}}=$ $\gamma$-aminobutyric acid $\mathrm{B} ; \mathrm{LTP}=$ long-term potentiation; $\mathrm{MCI}=$ mild cognitive impairment; $\mathrm{M} 1$ = primary motor cortex; $\mathrm{PAS}=$ paired associative stimulation; RAM = rapid alternating movement; TBI = traumatic brain injury; TMS = transcranial magnetic stimulation; $1 \mathrm{H}-\mathrm{MRS}=$ proton MR spectroscopy.
}

\section{Cognitive Function}

Cognitive Function in Young, Active Athletes With Multiple Concussions

Neuropsychological assessment, the gold standard in concussion diagnosis and management, outside the acute postinjury phase has revealed mixed results with respect to the long-term consequences of concussion in athletes. Iverson and colleagues ${ }^{36}$ found that high school athletes with a history of 1 or 2 prior concussions did not demonstrate any neuropsychological deficits 6 months after injury relative to athletes who had not suffered any concussions. Similarly, a study of collegiate athletes at least 6 months removed from their last injury did not have any detectable enduring neuropsychological changes. ${ }^{6}$ In contrast, a study of amateur soccer players showed that those with a history of concussion performed more poorly on neurocognitive tests. ${ }^{42}$ Authors of 2 other studies on the effects of multiple concussions in high school athletes found that those who had suffered 2 or more injuries had performance decrements on neurocognitive measures equivalent to the decrements of athletes in the acute postconcussion phase. ${ }^{50,51}$ Similarly, Collins and colleagues ${ }^{10}$ reported lowered baseline testing in college athletes with a history of concussion, although this was in conjunction with a learning disability, which may imply a detrimental synergistic relationship between concussions and impaired learning abilities. Another study revealed that professional soccer players, relative to elite noncontact sports athletes, demonstrated residual neuro- 


\section{De Beaumont, L. C. Henry, and N. Gosselin}

psychological deficits on memory, planning, and visuoperceptual tasks. ${ }^{43}$ Moreover, these deficits increased concurrently with the number of concussions and the frequency of heading the ball. The differential effects of heading and multiple concussions revealed disparate deficits on tests of visual and verbal memory and focused attention in the former, while sustained attention and visual processing were more affected in the latter. A study of jockeys demonstrated a parallel finding a minimum of 3 months after their most recent injury, with younger athletes more vulnerable to the effects of repeated concussions than their older counterparts. ${ }^{69}$ Along those lines, a recent consensus statement regarding the neuropsychological aspects of boxing strongly cautions against the potential neuropsychological sequelae of extended exposure to the sport. ${ }^{29}$ To this day, the exact nature of the cumulative effects of multiple concussions on neuropsychological function remains a subject of debate. What seems apparent is that there is a subgroup of athletes who are more vulnerable than others and that certain deficits are more conspicuous than others.

Why some studies have shown persistent neuropsychological deficits in concussed athletes and others have not is no small question to be swept aside. It stands to reason that before clinical manifestations (that is, reduced neurocognitive performance) become apparent, more sensitive neurophysiological measures of brain function could detect subclinical alterations left unnoticed with traditional neuropsychological testing. Neurophysiological measures (ERPs) as well as neuroimaging measures (1H-MRS, volumetric MRI, or calculation of cortical thickness) are powerful tools in the investigation of subclinical alterations due to multiple concussions.

Proton MRS has consistently shown alterations in concussed athletes in the acute, subacute, and chronic postinjury phases. ${ }^{7,30,31,33}$ Specifically, $N$-acetylaspartate is consistently diminished in concussed athletes relative to nonconcussed athletes. Other neurometabolic alterations include diminished levels of both glutamate, which seem to normalize by 6 months postinjury, and myoinositol, which may be elevated in the chronic postinjury phase..$^{30}$ However, no specific studies have assessed long-term neurometabolic changes in athletes who have sustained 1 versus multiple concussions. Diffusion tensor imaging is a noninvasive means of investigating the structural integrity of white matter by using the diffusive properties of water, and authors of several recent studies have attempted to identify white matter changes in the concussed brain by using this technology. ${ }^{15,30}$ Most studies have identified changes in fractional anisotropy following concussion, and several others have noted changes in mean diffusivity. The sum of the data at present suggests that there are notable significant changes in white matter integrity, although the persistence of these changes remains unknown. Perhaps these changes are transient and reflect postinjury edema. Conversely, these changes might be persistent and therefore characteristic of structural changes. The diffusion tensor imaging literature to date suggests some prospective validity in using the technique in diagnosis, but no specific brain areas hallmark the injury. Studies have revealed alterations in various tracts in the corpus callosum, cortical spinal tract, genu of the corpus callosum, posterior limb of the internal capsule, superior longitudinal fasciculus, and superior frontooccipital fasciculus to name a few. These alterations occur in part due to the highly variable nature of how concussions are sustained and in part due to individual factors. Diffusion tensor imaging studies have yet to focus on differences between athletes with single and multiple injuries, although Henry et al. ${ }^{30}$ found that the number of concussions did correlate with the number of brain regions showing diffusion alterations. A more recent technique, such as susceptibility-weighted imaging, has yet to be used enough for its efficacy to be assessed, although early evidence suggests that it may have utility in a pediatric population. ${ }^{44}$ It should be stressed that in all of the aforementioned techniques, the functional consequences for the metabolic or structural changes are unknown.

\section{Subclinical Alterations in Cognitive Function}

Event-related potentials are averaged electrical brain responses that allow one to determine the time course of higher level processes such as attention and memory updating in the human brain. ${ }^{54}$ The ERP measure represents the averaged electroencephalography signal time-locked to a presented stimulus and consists of different components labeled by their polarity (for example, $\mathrm{P}$ for positive and $\mathrm{N}$ for negative) and temporal range in milliseconds or by the ordinal number of major components. ${ }^{22}$ To date, most of the literature on ERPs and mild TBI has focused on the modulation of the classic P3 response as a result of concussion. The appeal of measuring the P3 component size and latency lies mostly in the ability to index changes in cognitive efficiency following a concussion. Evidence of enduring P3 changes in athletes who have suffered multiple concussions has abounded over the last decade. A P3 amplitude reduction exacerbated as a function of the number of previous concussions is the prevailing finding in young athletes with multiple concussions tested several months after their last injury. ${ }^{17,23,64}$ Authors of a more recent study reported persistent neurophysiological evidence of altered short-term memory retention in athletes with more than 3 concussions, as revealed by a significantly suppressed sustained posterior contralateral negativity waveform elicited by a visual short-term memory paradigm. ${ }^{65}$ Altered neurophysiological measures of performance monitoring was also found in athletes with multiple concussions, suggesting that recurrent concussions impair error processing mechanisms during cognitively engaging tasks. ${ }^{56}$ Taken together, these ERP findings suggest that multiple concussions pervasively alter neurophysiological indices of higher-order cognitive processing in young athletes who have long since returned to play.

Note, however, that very little clinical significance has been granted to ERP components alterations in concussed athletes, as the expert panel from the last symposium on sports concussions has yet to recognize ERPs as a useful tool for managing sports concussion cases. ${ }^{46}$ One of the main issues restraining the application of ERPs to current return-to-play protocols is the inability to relate these persistent ERP components alterations to either self-reported cognitive symptoms or behavioral performance alterations 
on validated neuropsychological measures of memory and attention functions beyond the acute postconcussion phase. Another major limitation is the relatively poor specificity of these ERP components, although they are highly sensitive to the effects of sports concussions. For instance, alterations in $\mathrm{P} 3$ components have been found in various clinical populations including those with schizophrenia, alcoholism, depression, attention deficit disorders, epilepsy, Alzheimer disease, and others. ${ }^{55}$ The poor ERP component specificity is particularly problematic for concussed athletes whose P3 components alterations are not tied to observable or reported cognitive difficulties, as one might argue that factors extraneous to the damaging impact of sports concussions on brain functions, such as concomitant depressive symptoms, attentional difficulties, or sleep disturbances, could just as well have influenced the P3 size and latency. Moreover, without supporting evidence from prospective studies focused on changes in ERP components relative to concussed athletes' own baseline measurements, we cannot determine whether these ERP components alterations are a consequence of sports concussions rather than a premorbid characteristic of athletes who are more at risk for sustaining sports concussions. Nevertheless, the consistent nature of ERP results across studies suggests that there may be subclinical alterations in athletes who have suffered multiple concussions.

\section{Cognitive Function in Late-Adulthood Athletes With Multiple Concussions}

While not typically disturbing daily life functions in young athletes, these subclinical alterations were linked to overt late-life clinical manifestations in a recent study conducted in late-adulthood athletes presenting with a history of remote concussions. ${ }^{20}$ Although not fully addressing the issue of cause and effect, evidence for the clinical significance of chronic P3 components alterations after sports concussions was revealed in a group of former athletes with concussions who had demonstrated reduced performance scores on neuropsychological tests that significantly correlated with $\mathrm{P} 3 \mathrm{a} / \mathrm{P} 3 \mathrm{~b}$ components amplitude attenuations. This finding confirms that concussion-related P3 components attenuations are associated with a worse performance on neuropsychological tests of episodic memory and executive function as athletes age and that the more the P3 components are suppressed, the greater the expected cognitive decline. ${ }^{20}$ The concept of "cognitive reserve" is very useful to help explain the resurgence of neuropsychological test performance decrements when athletes are tested more than 30 years postinjury. The central tenet of the cognitive reserve concept is that resilience to brain damage reflects how the brain uses its available resources to compensate for damage by optimizing performance through differential recruitment of brain networks and/or alternative cognitive strategies. ${ }^{2}$ Another important aspect of cognitive reserve is that damage from various environmental, developmental, and genotypic sources accumulates throughout a lifespan to reduce cognitive reserve. The progressive reduction in cognitive reserve via the accumulation of brain-damaging experiences and slowly waning health conditions throughout a lifespan characterizes the aging process and the accompanying de- cline in cognitive function..$^{13,14}$ When applied to concussion findings, evidence suggests that young concussed athletes who showed persistent P3 components alterations can rely on their cognitive reserve, thus allowing the recovery of baseline performance levels on neuropsychological tests after the acute postconcussion phase had subsided. Some $1 \mathrm{H}-\mathrm{MRS}^{32}$ evidence indicates continued metabolic alteration in asymptomatic athletes with concussed brain, while other data have shown that there is metabolic recovery. ${ }^{67,68}$ Although there is much work to be done to clarify if and/or how cognitive reserve plays a role in the compensation for and recovery from concussion, what seems more certain is that regardless of an asymptomatic state and a normal neurocognitive performance, subclinical differences persist in athletes who have sustained a concussion. Further, these differences are moderated by the number of concussions an athlete has sustained.

The picture becomes much clearer when older former athletes are considered. When the chronic deleterious effects of sports-related concussions on cognitive function were combined with known age-related cognitive decline, the ensuing cognitive reserve of formerly concussed athletes, relative to that of nonconcussed athletes, could no longer maintain optimal performance levels on neuropsychological tests of episodic memory and executive functions selected for their sensitivity to early symptoms of MCI. Note that MCI is known as a transitional stage between normal aging and dementia, including Alzheimer disease. Of great clinical relevance, former athletes with concussions performed significantly worse than their nonconcussed counterparts on cognitive functions that have recently been shown to be affected in the very early stages of MCI. ${ }^{3}$ Most notably, episodic memory decline in former athletes with concussion relative to controls was found in the recognition component of the Rey-Osterrieth Complex Figure Test, and scores on the immediate and delayed recall components also tended to be lower. Interestingly, visual recognition memory impairments were recently found early in the course of MCI. ${ }^{3}$ The acute sensitivity of visual recognition memory tests to MCI was related to the distribution of neurofibrillary tangles over hippocampal structures, which is known as a core neuropathological hallmark of Alzheimer disease. ${ }^{61}$ The recent demonstration of strong correlations between visual recognition memory decline and 1H-MRS/volumetric MRI evidence of compromised hippocampal formation integrity in former athletes in late adulthood with remote concussions $^{66}$ highlights important similarities between the pathophysiology of sports concussions and that of MCIs. However, the presence of widespread cortical thinning together with elevated choline levels in dorsolateral prefrontal cortices not typically found in patients with MCI suggests that sports concussion in aging athletes exerts its own pathophysiological signature. ${ }^{66}$

\section{Motor Function}

Motor Function in Young, Active Athletes With Multiple Concussions

While the recovery of cognitive impairments after sports concussions has drawn most of the attention from 


\section{De Beaumont, L. C. Henry, and N. Gosselin}

the scientific community over the last few decades, the investigation of motor system abnormalities has recently come to the forefront of the sports concussion literature. Indeed, assessment of postural stability with various stances and surfaces was recently integrated into clinical practice to assist clinicians in determining when concussed athletes who experienced balance problems after injury could safely return to play. ${ }^{28}$ While postural stability has typically been found to return to baseline levels within a few days postconcussion on conventional measures of COP displacement, ${ }^{8,9,45}$ the recent addition of approximate entropy calculation as a nonlinear dynamic measure of postural control has revealed increased sensitivity to subtle physiological alterations associated with sports concussions. ${ }^{9}$ Indeed, athletes who resumed competition more than 9 months prior to testing still exhibited enhanced COP oscillation regularity according to an approximate entropy measure of postural control despite normal postural stability scores on conventional linear measures. Although the functional significance of enhanced COP oscillation regularity with regard to postural stability is still largely unknown, previous studies have suggested that it may represent an adaptive compensatory mechanism put forth by concussed athletes to achieve postural stability. ${ }^{8,25}$ Another possibility is that concussive injuries result in stiffened lower extremity musculature potentially related to excessive motor system excitability after sports concussions.

The application of TMS for sports concussion emerged mostly from postural imbalance data possibly involving motor system excitability abnormalities coupled with the TMS demonstration of altered cortical excitability of the M1 in the acute postconcussion phase. ${ }^{18}$ This relatively novel technique has been reliably used to detect preclinical covert indices of motor disorders. ${ }^{38}$ Among primary TMS measures, the CSP revealed unprecedented sensitivity to the subtle alterations in central inhibitory/ excitatory mechanisms of the motor system in concussed athletes. ${ }^{1}$ The later part of the CSP has been attributed to activity of $\mathrm{GABA}_{\mathrm{B}}$-mediated intracortical inhibitory systems of the M1, whereas spinal inhibition contributes to its early part. ${ }^{34}$ Increased intracortical inhibition of M1, as reflected by prolonged CSP duration, was found in concussed athletes who had been asymptomatic on average 2 years prior to testing. ${ }^{18}$ In an earlier study, we prospectively examined the effects of recurrent concussions in a small group of athletes with multiple concussions who were retested using the same experimental protocol and found that the CSP was further prolonged after another concussion was sustained, suggesting that M1 intracortical inhibitory receptors may be particularly vulnerable to the effects of subsequent concussions. ${ }^{18}$ Based on these findings, it would appear that concussions alter the efficacy of $\mathrm{GABA}_{\mathrm{B}}$ receptor systems, perhaps contributing to rendering the brain more vulnerable to subsequent traumatic events. The discovery of lifelong $\mathrm{GABA}_{\mathrm{B}}$-mediated intracortical inhibition alterations of the $\mathrm{M} 1$ gave rise to extensive ramifications into future clinical studies of sports concussions on associated functional losses and potential treatment alternatives.

One important discovery derived from alterations in intracortical inhibitory mechanisms in concussed athletes was inspired by pharmacological evidence suggesting that $\mathrm{GABA}_{\mathrm{B}}$ receptors play an important role in $\mathrm{LTP}^{16}$ and learning. ${ }^{49}$ Briefly, it is well known that synaptic plasticity occurring through LTP is an essential part of motor learning. In animal preparations, motor learning strengthens M1 synaptic efficacy ${ }^{60}$ and prevents subsequent LTP $^{59}$ from occurring without affecting synaptic modification range, ${ }^{59}$ which, according to the Bienenstock-Cooper-Munro theory of bidirectional synaptic plasticity, ${ }^{5}$ is indicative of LTP involvement in motor skill learning. Recent advances in TMS research have enabled the noninvasive induction of LTP in human M1 to study its role in skill acquisition. In PAS paradigms, stimulation of the median nerve is repetitively paired with TMS of its homotopic representation in the M1, ${ }^{63}$ resulting in increased motor cortex excitability. Paired associative stimulation induction of LTP-like effects are reversible, topographically specific, and dependent on $N$-methyl-D-aspartate receptors. ${ }^{62,63}$ Similar to what has been shown in animals, ${ }^{59}$ a period of motor learning prevents LTP-like plasticity from occurring after PAS, ${ }^{70}$ suggesting that motor learning occurs more easily in a system in which LTP induction is easily achieved. Knowing that the $\mathrm{GABA}_{\mathrm{B}}$ receptor agonist baclofen abolishes PASinduced LTP-like plasticity, ${ }^{47}$ some have proposed that increases in $\mathrm{GABA}_{\mathrm{B}}$ receptor activity prevent LTP-dependent motor learning. ${ }^{47}$ Therefore, it stands to reason that excessive $\mathrm{GABA}_{\mathrm{B}}$ neurotransmission in the motor cortex of concussed athletes may reduce the probability of LTP induction, resulting in impaired motor learning. Recent findings consistent with this notion indicate that repeated concussions induced persistent elevations in $\mathrm{GABA}_{\mathrm{B}}$-mediated intracortical inhibition in the M1, which was associated with suppressed PAS-induced LTP-like synaptic plasticity. ${ }^{21}$ This synaptic plasticity suppression was related to reduced motor learning relative to normal LTP-like synaptic plasticity in nonconcussed players. These findings reveal GABA neurotransmission alterations after repeated concussions and suggest that impaired learning in athletes with multiple concussions could at least partly be related to compromised GABA-dependent LTP synaptic plasticity. ${ }^{21}$

\section{Motor Function in Late-Adulthood Athletes With Multiple Concussions}

Consistent with previous findings in young athletes who have suffered multiple concussions, TMS assessment of motor cortex excitability showed that the CSP was significantly prolonged in former athletes who had sustained their last sports concussion more than 3 decades prior to testing. ${ }^{20}$ Along with their prolonged CSP relative to controls, former athletes with a history of sports concussion exhibited significant slowness of movement, or bradykinesia, on an RAM task. Further correlational analyses established a strong relationship between the duration of the CSP and the movement velocity on the RAM task, such that former concussed athletes with more prolonged CSPs tended to be slower at executing alternating pronation-supination cycles. This finding suggests that the altered neurophysiological mechanisms that lengthen the CSP in concussed athletes could also be implicated in the biological bases of the slowness of movement seen 
in former athletes with concussion when performing an RAM task. ${ }^{20}$

This significant slowness in executing RAM task cycles was found in former athletes with concussion who did not otherwise report experiencing motor difficulties in their daily activities. This finding is consistent with a recent study showing motor slowness on an RAM task in patients with very-early-stage Parkinson disease who had yet to experience the degenerative disease's more debilitating symptoms.$^{39}$ It remains to be verified in further longitudinal studies whether former concussed athletes who experienced early signs of movement slowness at the time of testing will proceed to incapacitating motor symptoms. Interestingly, motor execution speed on an identical RAM task was normal in young, active university football players with multiple concussions. ${ }^{19}$ The concept of cognitive reserve discussed earlier to explain the reappearance of neuropsychological test deficits in former athletes with concussions relative to nonconcussed counterparts could apply just as well to bradykinesia symptoms surfacing 3 decades postconcussion. In fact, the resilience of the young concussed brain in adapting to abnormal intracortical inhibition of the motor cortex appears to be sufficient to maintain normal motor execution speed, whereas the nature of an aging brain that had sustained prior concussions could no longer enable optimal performance levels.

Besides the depleted neural reserve, collected data cannot exclude the possibility that former concussed athletes were experiencing early symptoms of a neurodegenerative disease affecting motor system functions. This finding is in keeping with the catastrophic consequences of repetitive subconcussive and concussive head blows to the long-term motor system function of boxers. ${ }^{57}$ In fact, abnormal performance on memory tests, increased motor and speech latencies, dysarthria, pyramidal tract dysfunction, tremor in the head and upper extremities, and behavioral changes are common features associated with what has often been referred to as "dementia pugilistica," or chronic TBI. ${ }^{57}$ Of great clinical relevance, motor symptoms of chronic TBI are typically the earliest clinical manifestations of this neurodegenerative condition. ${ }^{57}$ Among the cardinal motor impairments of chronic TBI, ataxia and motor execution slowness (bradykinesia) have both been related to significantly prolonged CSP duration in other neurodegenerative conditions. In fact, previous studies conducted in patients with cerebellar ataxia ${ }^{58}$ and Huntington disease ${ }^{40}$ have also revealed excessive CSP alongside bradykinesia/ataxia symptoms. Investigating the effects of chronic TBI relative to those of fewer remote concussions on the $\mathrm{GABA}_{\mathrm{B}}$-mediated intracortical inhibition anomalies and associated motor symptoms could also help to establish the clinical significance of lifelong CSP anomalies after sports concussions.

\section{Conclusions}

In summary, the research to date has consistently shown enduring, cumulative cognitive and motor system function alterations after sports concussions that are present in both young concussed athletes and older former athletes who had previously sustained concussions.
Strong converging evidence outlined in this review indicated that the damaging effects of concussion cannot and should not be overlooked any longer. Further, research efforts should be increased to deepen our understanding of the long-term pathophysiology of sports concussions and how it might be influenced by genetic and environmental factors. Additionally, investigating how more proactive injury management might influence the long-term and/or cumulative effects of the injury must be explored. Furthermore, we also highlighted the usefulness of more refined brain investigation tools to delineate the underlying causes and associated action mechanisms preventing the brain from fully recovering from the seemingly benign adverse effects of sports concussions, even if the persistent changes are subclinical. Indeed, the use of refined brain investigation tools is especially called for if we are to move from current return-to-play management based mostly on expert opinion to an evidence-based medical approach. We also focused on the importance of implementing longitudinal follow-up studies of former concussed athletes, whose cognitive and motor system functional alterations may evolve into more severe, perhaps debilitating difficulties impairing daily function.

\section{Disclosure}

The authors report no conflict of interest concerning the materials or methods used in this study or the findings specified in this paper.

Author contributions to the study and manuscript preparation include the following. Conception and design: De Beaumont. Drafting the article: De Beaumont. Critically revising the article: Henry, Gosselin. Reviewed submitted version of manuscript: Henry, Gosselin. Approved the final version of the manuscript on behalf of all authors: De Beaumont.

\section{References}

1. Abbruzzese G, Trompetto C: Clinical and research methods for evaluating cortical excitability. J Clin Neurophysiol 19:307321,2002

2. Allen JS, Bruss J, Damasio H: The aging brain: the cognitive reserve hypothesis and hominid evolution. Am J Hum Biol 17:673-689, 2005

3. Barbeau E, Didic M, Tramoni E, Felician O, Joubert S, Sontheimer A, et al: Evaluation of visual recognition memory in MCI patients. Neurology 62:1317-1322, 2004

4. Bernstein DM: Information processing difficulty long after self-reported concussion. J Int Neuropsychol Soc 8:673682,2002

5. Bienenstock EL, Cooper LN, Munro PW: Theory for the development of neuron selectivity: orientation specificity and binocular interaction in visual cortex. J Neurosci 2:32-48, 1982

6. Bruce JM, Echemendia RJ: History of multiple self-reported concussions is not associated with reduced cognitive abilities. Neurosurgery 64:100-106, 2009

7. Carrillo C, Prarat M, Vagnozzi A, Calahan JD, Smoliga G, Nelson WM, et al: Specific detection of Rinderpest virus by real-time reverse transcription-PCR in preclinical and clinical samples from experimentally infected cattle. J Clin Microbiol 48:4094-4101, 2010

8. Cavanaugh JT, Guskiewicz KM, Giuliani C, Marshall S, Mercer VS, Stergiou N: Recovery of postural control after cerebral concussion: new insights using approximate entropy. J Athl Train 41:305-313, 2006

9. Cavanaugh JT, Guskiewicz KM, Stergiou N: A nonlinear dy- 
namic approach for evaluating postural control: new directions for the management of sport-related cerebral concussion. Sports Med 35:935-950, 2005

10. Collins MW, Grindel SH, Lovell MR, Dede DE, Moser DJ, Phalin BR, et al: Relationship between concussion and neuropsychological performance in college football players. JAMA 282:964-970, 1999

11. Collins MW, Lovell MR, Iverson GL, Cantu RC, Maroon JC, Field M: Cumulative effects of concussion in high school athletes. Neurosurgery 51:1175-1181, 2002

12. Corsellis JA, Bruton CJ, Freeman-Browne D: The aftermath of boxing. Psychol Med 3:270-303, 1973

13. Craik FI, Bialystok E: Cognition through the lifespan: mechanisms of change. Trends Cogn Sci 10:131-138, 2006

14. Craik FI, Salthouse TA (eds): The Handbook of Aging and Cognition, ed 2. Mahwah, NJ: Lawrence Erlbaum Associates, 1999

15. Cubon VA, Putukian M, Boyer C, Dettwiler A: A diffusion tensor imaging study on the white matter skeleton in individuals with sports-related concussion. J Neurotrauma 28:189201, 2011

16. Davies CH, Starkey SJ, Pozza MF, Collingridge GL: GABA autoreceptors regulate the induction of LTP. Nature 349:609611, 1991

17. De Beaumont L, Brisson B, Lassonde M, Jolicoeur P: Longterm electrophysiological changes in athletes with a history of multiple concussions. Brain Inj 21:631-644, 2007

18. De Beaumont L, Lassonde M, Leclerc S, Théoret H: Longterm and cumulative effects of sports concussion on motor cortex inhibition. Neurosurgery 61:329-337, 2007

19. De Beaumont L, Mongeon D, Tremblay S, Messier J, Prince F, Leclerc S, et al: Persistent motor system abnormalities in formerly concussed athletes. J Athl Train 46:234-240, 2011

20. De Beaumont L, Théoret H, Mongeon D, Messier J, Leclerc S, Tremblay S, et al: Brain function decline in healthy retired athletes who sustained their last sports concussion in early adulthood. Brain 132:695-708, 2009

21. De Beaumont L, Tremblay S, Poirier J, Lassonde M, Théoret $\mathrm{H}$ : Altered bidirectional plasticity and reduced implicit motor learning in concussed athletes. Cereb Cortex 22:112-121, 2012

22. Donchin E: A multivariate approach to the analysis of average evoked potentials. IEEE Trans Biomed Eng 13:131-139, 1966

23. Gaetz M, Goodman D, Weinberg H: Electrophysiological evidence for the cumulative effects of concussion. Brain Inj 14: 1077-1088, 2000

24. Gerberich SG, Priest JD, Boen JR, Straub CP, Maxwell RE: Concussion incidences and severity in secondary school varsity football players. Am J Public Health 73:1370-1375, 1983

25. Geurts AC, de Haart M, van Nes IJ, Duysens J: A review of standing balance recovery from stroke. Gait Posture 22:267281,2005

26. Gosselin N, Thériault M, Leclerc S, Montplaisir J, Lassonde M: Neurophysiological anomalies in symptomatic and asymptomatic concussed athletes. Neurosurgery 58:1151-1161, 2006

27. Guskiewicz KM, McCrea M, Marshall SW, Cantu RC, Randolph C, Barr W, et al: Cumulative effects associated with recurrent concussion in collegiate football players: the NCAA Concussion Study. JAMA 290:2549-2555, 2003

28. Guskiewicz KM, Perrin DH, Gansneder BM: Effect of mild head injury on postural stability in athletes. J Athl Train 31: 300-306, 1996

29. Heilbronner RL, Bush SS, Ravdin LD, Barth JT, Iverson GL, Ruff RM, et al: Neuropsychological consequences of boxing and recommendations to improve safety: a National Academy of Neuropsychology education paper. Arch Clin Neuropsychol 24:11-19, 2009

30. Henry LC, Tremblay J, Tremblay S, Lee A, Brun C, Lepore
$\mathrm{N}$, et al: Acute and chronic changes in diffusivity measures after sports concussion. J Neurotrauma 28:2049-2059, 2011

31. Henry LC, Tremblay S, Boulanger Y, Ellemberg D, Lassonde M: Neurometabolic changes in the acute phase after sports concussions correlate with symptom severity. J Neurotrauma 27:65-76, 2010

32. Henry LC, Tremblay S, Leclerc S, Khiat A, Boulanger Y, Ellemberg D, et al: Metabolic changes in concussed American football players during the acute and chronic post-injury phases. BMC Neurol 11:105, 2011

33. Hollister JR, Vagnozzi A, Knowles NJ, Rieder E: Molecular and phylogenetic analyses of bovine rhinovirus type 2 shows it is closely related to foot-and-mouth disease virus. Virology 373:411-425, 2008

34. Inghilleri M, Berardelli A, Cruccu G, Manfredi M: Silent period evoked by transcranial stimulation of the human cortex and cervicomedullary junction. J Physiol 466:521-534, 1993

35. Iverson GL: Outcome from mild traumatic brain injury. Curr Opin Psychiatry 18:301-317, 2005

36. Iverson GL, Brooks BL, Collins MW, Lovell MR: Tracking neuropsychological recovery following concussion in sport. Brain Inj 20:245-252, 2006

37. Iverson GL, Gaetz M, Lovell MR, Collins MW: Cumulative effects of concussion in amateur athletes. Brain Inj 18:433443, 2004

38. Kobayashi M, Pascual-Leone A: Transcranial magnetic stimulation in neurology. Lancet Neurol 2:145-156, 2003

39. Koop MM, Shivitz N, Brontë-Stewart H: Quantitative measures of fine motor, limb, and postural bradykinesia in very early stage, untreated Parkinson's disease. Mov Disord 23:12621268,2008

40. Lefaucheur JP, Ménard-Lefaucheur I, Maison P, Baudic S, Cesaro P, Peschanski M, et al: Electrophysiological deterioration over time in patients with Huntington's disease. Mov Disord 21:1350-1354, 2006

41. Macciocchi SN, Barth JT, Littlefield L, Cantu RC: Multiple concussions and neuropsychological functioning in collegiate football players. J Athl Train 36:303-306, 2001

42. Matser EJ, Kessels AG, Lezak MD, Jordan BD, Troost J: Neuropsychological impairment in amateur soccer players. JAMA 282: 971-973, 1999

43. Matser JT, Kessels AG, Jordan BD, Lezak MD, Troost J: Chronic traumatic brain injury in professional soccer players. Neurology 51:791-796, 1998

44. Maugans TA, Farley C, Altaye M, Leach J, Cecil KM: Pediatric sports-related concussion produces cerebral blood flow alterations. Pediatrics 129:28-37, 2012

45. McCrea M, Guskiewicz KM, Marshall SW, Barr W, Randolph C, Cantu RC, et al: Acute effects and recovery time following concussion in collegiate football players: the NCAA Concussion Study. JAMA 290:2556-2563, 2003

46. McCrory PR, Johnston K, Meeuwisse W, Aubry M, Cantu $\mathrm{RC}$, Dvorak J, et al: Summary and agreement statement of the 2nd International Conference on Concussion in Sport, Prague 2004. Clin J Sport Med 15:48-55, 2005

47. McDonnell MN, Orekhov Y, Ziemann U: Suppression of LTPlike plasticity in human motor cortex by the GABAB receptor agonist baclofen. Exp Brain Res 180:181-186, 2007

48. McKee AC, Cantu RC, Nowinski CJ, Hedley-Whyte ET, Gavett BE, Budson AE, et al: Chronic traumatic encephalopathy in athletes: progressive tauopathy after repetitive head injury. J Neuropathol Exp Neurol 68:709-735, 2009

49. McNamara RK, Skelton RW: Baclofen, a selective GABAB receptor agonist, dose-dependently impairs spatial learning in rats. Pharmacol Biochem Behav 53:303-308, 1996

50. Moser RS, Schatz P: Enduring effects of concussion in youth athletes. Arch Clin Neuropsychol 17:91-100, 2002

51. Moser RS, Schatz P, Jordan BD: Prolonged effects of concussion in high school athletes. Neurosurgery 57:300-306, 2005

52. Omalu BI, DeKosky ST, Minster RL, Kamboh MI, Hamil- 


\section{Enduring alterations in sports concussions}

ton RL, Wecht CH: Chronic traumatic encephalopathy in a National Football League player. Neurosurgery 57:128-134, 2005

53. Petersen RC: Mild cognitive impairment as a diagnostic entity. J Intern Med 256:183-194, 2004

54. Polich J: Bifurcated P300 peaks: P3a and P3b revisited? J Clin Neurophysiol 5:287-294, 1988

55. Polich J: Clinical application of the P300 event-related brain potential. Phys Med Rehabil Clin N Am 15:133-161, 2004

56. Pontifex MB, O'Connor PM, Broglio SP, Hillman CH: The association between mild traumatic brain injury history and cognitive control. Neuropsychologia 47:3210-3216, 2009

57. Rabadi MH, Jordan BD: The cumulative effect of repetitive concussion in sports. Clin J Sport Med 11:194-198, 2001

58. Restivo DA, Lanza S, Giuffrida S, Le Pira F, Drago MT, Di Mauro R, et al: Cortical silent period prolongation in spinocerebellar ataxia type 2 (SCA2). Funct Neurol 19:37-41, 2004 (Erratum in Funct Neurol 19:preceding table of contents, 2004)

59. Rioult-Pedotti MS, Friedman D, Donoghue JP: Learning-induced LTP in neocortex. Science 290:533-536, 2000

60. Rioult-Pedotti MS, Friedman D, Hess G, Donoghue JP: Strengthening of horizontal cortical connections following skill learning. Nat Neurosci 1:230-234, 1998

61. Selkoe DJ: Alzheimer's disease. In the beginning.... Nature 354:432-433, 1991

62. Stefan K, Kunesch E, Benecke R, Cohen LG, Classen J: Mechanisms of enhancement of human motor cortex excitability induced by interventional paired associative stimulation. J Physiol 543:699-708, 2002

63. Stefan K, Kunesch E, Cohen LG, Benecke R, Classen J: Induction of plasticity in the human motor cortex by paired associative stimulation. Brain 123:572-584, 2000

64. Thériault M, De Beaumont L, Gosselin N, Filipinni M, Lassonde M: Electrophysiological abnormalities in well functioning multiple concussed athletes. Brain Inj 23:899-906, 2009

65. Thériault M, De Beaumont L, Tremblay S, Lassonde M, Jolicoeur P: Cumulative effects of concussions in athletes re- vealed by electrophysiological abnormalities on visual working memory. J Clin Exp Neuropsychol 33:30-41, 2011

66. Tremblay S, De Beaumont L, Henry LC, Boulanger Y, Evans $\mathrm{AC}$, Bourgouin P, et al: Sports concussions and aging: a neuroimaging investigation. Cereb Cortex, 2012

67. Vagnozzi R, Signoretti S, Cristofori L, Alessandrini F, Floris $\mathrm{R}$, Isgro $\mathrm{E}$, et al: Assessment of metabolic brain damage and recovery following mild traumatic brain injury: a multicentre, proton magnetic resonance spectroscopic study in concussed patients. Brain:3232-3242, 2010

68. Vagnozzi R, Signoretti S, Tavazzi B, Floris R, Ludovici A, Marziali S, et al: Temporal window of metabolic brain vulnerability to concussion: a pilot $1 \mathrm{H}$-magnetic resonance spectroscopic study in concussed athletes-part III. Neurosurgery 62:1286-1296, 2008

69. Wall SE, Williams WH, Cartwright-Hatton S, Kelly TP, Murray J, Murray M, et al: Neuropsychological dysfunction following repeat concussions in jockeys. J Neurol Neurosurg Psychiatry 77:518-520, 2006

70. Ziemann U, Ilić TV, Pauli C, Meintzschel F, Ruge D: Learning modifies subsequent induction of long-term potentiation-like and long-term depression-like plasticity in human motor cortex. J Neurosci 24:1666-1672, 2004 (Erratum in J Neurosci 24:1 $\mathrm{p}$ following 10552,2004 )

71. Zillmer EA, Spiers MV, Culbertson WC: Principles of Neuropsychology. Belmont, CA: Wadsworth, 2001

Manuscript submitted August 6, 2012.

Accepted September 27, 2012.

Please include this information when citing this paper: DOI: 10.3171/2012.9.FOCUS12278.

Address correspondence to: Louis De Beaumont, Ph.D., Department of Psychology, Université du Québec à Trois-Rivières, MichelSarrazin Hall, C.P. 500, Trois-Rivières, Québec G9A 5H7, Canada. email: louis.de.beaumont@umontreal.ca. 